\title{
ELLIPTICALLY EMBEDDED SUBGROUPS OF POLYCYCLIC GROUPS
}

\author{
A. H. RHEMTULLA AND J. S. WILSON
}

(Communicated by Bhama Srinivasam)

\begin{abstract}
A subgroup $H$ of a group $G$ is elliptically embedded in $G$ if for each subgroup $K$ of $G$ there is an integer $n=n(K)$ such that $\langle H, K\rangle=H K \cdots H K$, where the product has $2 n$ factors. It is shown that a subgroup $H$ of a polycyclic by finite group $G$ is elliptically embedded in $G$ if and only if $H$ is subnormal in some subgroup of finite index in $G$.
\end{abstract}

1. Introduction. Let $H$ be a subgroup of a group $G$. We say that $H$ is elliptically embedded in $G$, and we write $H$ ee $G$, if for each subgroup $K$ of $G$ there is an integer $n$ depending on $K$ such that

$$
\langle H, K\rangle=H K \cdots H K
$$

where the product has $2 n$ factors. Elliptically embedded subgroups were first studied in [2], and a number of connections between subnormality and the property of being elliptically embedded were established. In particular, the results of [2] imply that every subnormal subgroup of a polycyclic group is elliptically embedded and that every cyclic elliptically embedded subgroup of a torsion-free soluble group is subnormal. While the former of these results follows from an easy induction, the latter lies rather deeper, and depends ultimately on some delicate calculations with complex numbers of bounded modulus. In this note we use a recent and very deep result in number theory, proved by Van der Poorten [3] and Evertse [1], to give a characterization of the elliptically embedded subgroups of polycyclic by finite groups.

THEOREM. Let $H$ be a subgroup of a polycyclic by finite group $G$. Then $H$ is elliptically embedded in $G$ if and only if $H$ is subnormal in some subgroup of finite index in $G$.

2. We begin by proving an elementary result.

LEMMA 1. If $H \leq G$ and $\left|G: N_{G}(H)\right|$ is finite then $H$ ee $G$.

ProOF. It suffices to show that if $G$ is a group generated by subgroups $H, K$ such that $\left|G: N_{G}(H)\right|$ is finite then $G=(H K)^{n}$ for some integer $n$, where $(H K)^{n}$ stands for the product $H K \cdots H K$ with $2 n$ factors. Write $N=N_{G}(H)$ and $M=$ $\bigcap\left(N^{g} ; g \in G\right)$. Thus $H \cap M \triangleleft M \triangleleft G$ and $G / M$ is finite. Each of the finitely many conjugates of $H \cap M$ is normal in $M$, and so the normal closure $L$ of $H \cap M$ in

Received by the editors June 9, 1986 and, in revised form, December 26, 1986.

1980 Mathematics Subject Classification (1985 Revision). Primary 20F26; Secondary 20E15, $20 \mathrm{~F} 16$.

Key words and phrases. Polycyclic groups, subnormal subgroups.

(C) 1988 American Mathematical Society $0002-9939 / 88 \$ 1.00+\$ .25$ per page 
$G$ is their product. It follows that $L \leq(H K)^{n}$ for some $n$. Therefore there is no harm in assuming that $L=1$. Thus $H \cap M=1$ and $H$ is finite. Since $\left|G: N_{G}(H)\right|$ is finite, it follows that the normal closure $H^{G}$ of $H$ in $G$ is finitely generated and lies in the $F C$-centre of $G$. Thus $H^{G}$ is centre by finite and hence finite by abelian; and because it is generated by the finitely many conjugates of $H$, it must in fact be finite. However $G=\left(H^{G}\right) K$, and the result follows.

We can now prove one of the implications in the Theorem. Suppose that $G$ is polycyclic by finite and that $H$ is subnormal in a subgroup of finite index in $G$. Let $K \leq G$ and let $G_{1}=\langle H, K\rangle$. There is a chain of subgroups

$$
H=H_{d} \triangleleft \cdots \triangleleft H_{1} \triangleleft H_{0} \leq G_{1}
$$

with $\left|G_{1}: H_{0}\right|$ finite, and we prove by induction on $d$ that $G_{1}=(H K)^{n}$ for some integer $n$. If $d \leq 1$ this follows from Lemma 1. If $d>1$, let $L$ be the normal closure of $H$ in $H_{d-2}$. Each conjugate of $H$ in $H_{d-2}$ is normal in $L$ since

$$
L \leq H_{d-1}^{H_{d-2}} \leq H_{d-1} .
$$

Thus since $G_{1}$ is polycyclic by finite $L$ is a product of finitely many conjugates of $H$, and so

$$
L \leq(H K)^{r}
$$

for some integer $r$. However $L \triangleleft H_{d-2}$ and $G_{1}=\langle L, K\rangle$, so by induction we have

$$
G_{1}=(L K)^{s}
$$

for some integer $s$. The result follows on combining (1) and (2).

3. For brevity, we write $L \operatorname{snf} M$ to mean that $L$ is a subnormal subgroup of a subgroup of finite index in the group $M$. Before considering the other implication in the Theorem we prove that if $H$ is a subgroup of a polycyclic by finite group $G$ and if there is a chain of subgroups

$$
H=H_{n} \leq \cdots \leq H_{0}=G
$$

such that for each $i$ either $H_{i} \triangleleft H_{i-1}$ or $\left|H_{i-1}: H_{i}\right|$ is finite, then $H$ snf $G$. If $n \leq 1$ this is clear. If $n>1$ we may suppose by induction that $H$ is subnormal in a subgroup $L$ of finite index $e$ in $H_{1}$. If $\left|G: H_{1}\right|$ is finite, the result follows. Otherwise both $H_{1}$ and $R=\left\langle x^{e} ; x \in H_{1}\right\rangle$ are normal in $G$, and the centralizer $C$ in $G$ of $H_{1} / R$ has finite index in $G$ since $H_{1} / R$ is finite. Because $R \leq L \leq H_{1}$, we have $[L, C] \leq R \leq L$, so that $L \triangleleft L C$. Thus $L C$ is a subgroup of finite index in which $H$ is subnormal.

Using this observation we can now reduce the proof of the other implication in the Theorem to the proof of the following Lemma.

LEMMA 2. Let $A$ be a free abelian group of finite rank and let $H$ be a polycyclic by finite group which acts rationally irreducibly on $A$. If $H$ is elliptically embedded in the split extension of $A$ by $H$ then $H$ acts trivially on $A$.

Let $G$ be polycyclic by finite and let $H$ ee $G$. We show by induction on the Hirsch number of $G$ that $H \operatorname{snf} G$. If $G$ is finite, this is obvious. Otherwise $G$ has a 
free abelian normal subgroup $B \neq 1$. Clearly $H B / B$ ee $G / B$, and so by induction $H B \operatorname{snf} G$. If we can prove that $H$ snf $H B$ then it will follow from above that $H$ snf $G$. Let $A$ be a nontrivial subgroup of $B$ of minimal rank with respect to being normalized by $H$. Thus $A \triangleleft H B$ and induction yields that $H A$ snf $H B$. Therefore it will suffice to show that $H \operatorname{snf} H A$. If $H \cap A \neq 1$ then, being normalized by $H$, $H \cap A$ has finite index in $A$ so that $|H A: H|$ is actually finite. Otherwise $H A$ is a split extension of $A$ by $H$, and $H \triangleleft H A$ by Lemma 2 .

4. The following result was proved as Theorem 1 of [3], and a very similar result appears as Theorem 1 of [1].

TheOREM (VAN DER POORTEN AND EVERTSE). Let $K$ be a field of characteristic zero and $H$ a finitely generated subgroup of the multiplicative group of $K$. Then for each integer $m>0$ there are only finitely many essentially distinct relations

$$
u_{1}+u_{2}+\cdots+u_{m}=1
$$

with each $u_{i}$ in $H$.

Here, two relations are regarded as essentially distinct if they do not differ by vanishing subsums of elements of $H$. Using this Theorem we now prove Lemma 2 .

Suppose Lemma 2 false. Since $C_{H}(A) \triangleleft G$, we may assume that $H$ acts faithfully on $A$. Thus since $A$ is free abelian of finite rank, $H$ has an abelian normal subgroup $K$ of finite index. Application of Clifford's Theorem to the irreducible $Q H$-module $A \otimes_{Z} Q$ shows that there exists $a \in A \backslash 0$ and there exist elements $t_{1}=1, t_{2}, \ldots, t_{r}$ from distinct cosets of $K$ in $H$ such that

(i) $B_{i}=\left(a t_{i}\right) Z K$ is a rationally irreducible $Z K$-module for $i=1, \ldots, r$,

(ii) the sum $A_{0}$ of $B_{1}, \ldots, B_{r}$ is their direct sum, and

(iii) $A / A_{0}$ is finite.

Let $\left\{t_{1}, \ldots, t_{s}\right\}$ be a transversal to $K$ in $H$. By (iii) there is an integer $z>0$ such that $z a t_{j} \in A_{0}$ for each $j>r$; thus each such $z a t_{j}$ is a $Z$-linear combination of elements $a t_{i} k$ with $i \leq r$ and $k \in K$, and there is an integer $l>0$ such that each $z a t_{j}$ with $j>r$ is a sum of at most $l$ elements of the form $\pm a t_{i} k$ with $i \leq r$ and $k \in K$. It follows that each element $z a h$ with $h \in H$ is a sum of at most $m$ elements $\pm a t_{i} k$ with $i \leq r$ and $k \in K$, where $m=\max \{z, l\}$.

If $\left\langle H, H^{a}\right\rangle=H$ then $[a, h] \in A \cap H=1$ for each $h \in H$ so that $C_{A}(H)$ is a nontrivial $H$-invariant subgroup of $A$, and a contradiction ensues. Writing $D=A \cap\left\langle H, H^{a}\right\rangle$, we therefore have

$$
H<\left\langle H, H^{a}\right\rangle=H D,
$$

so that $D \neq 1$. Thus since $H$ acts rationally irreducibly on $A$ and since $D$ is a $H$-invariant subgroup, $D$ has finite index in $A$. Because $H$ ee $G$ there is an integer $n$ such that each element of $\left\langle H, H^{a}\right\rangle$ is a product of $n$ terms of the form

$$
h_{1} h_{2}^{a}=h_{1} a^{-1} h_{2} a
$$

with $h_{1}, h_{2} \in H$. Collecting the elements of $H$ in such a product on the left, we see that each element of $\left\langle H, H^{a}\right\rangle$ is a product of an element of $H$ and $2 n$ conjugates of $a^{ \pm 1}$ under the elements of $H$. Thus each element of $D$ is, in additive notation, 
a sum of $2 n$ terms of the form $\pm a h$ with $h \in H$, and so from the above paragraph each element of $z D$ can be written as a sum of at most $n_{1}$ terms $\pm a t_{i} k$ with $i \leq r$ and $k \in K$, where $n_{1}=2 n m$. Since the sum of the subgroups $B_{1}, \ldots, B_{r}$ is direct we conclude, on equating coefficients in $B_{1}$, that each element of $z D \cap B_{1}$ is a sum of at most $n_{1}$ terms $\pm a k$ with $k \in K$.

Because $B_{1}$ is a rationally irreducible $Z K$-module, $V=B_{1} \otimes_{Z} Q$ is an irreducible $Q K$-module, and so by Schur's Lemma the centralizer ring $\Gamma=\operatorname{End}_{Q K}(V)$ is a division ring finite-dimensional over $Q$. Since $K$ is abelian, its image $\bar{K}$ in $\operatorname{End}_{Q}(V)$ lies in and spans $\Gamma$, so that $\Gamma$ is an algebraic number field. Further, regarded as a $\Gamma$-vector space, $V$ must be one dimensional. Since $D$ has finite index in $A$, the subgroup $(z D) \cap B_{1}$ is nontrivial. Let $a \delta \in(z D) \cap B_{1}$ with $\delta \in \Gamma \backslash 0$. Thus $m \delta$ is a sum of at most $n_{1}$ terms $\pm k$ with $k \in \bar{K}$, for each $m \in Z$, and each integer $m$ is a sum of at most $n_{1}$ elements of the finitely generated multiplicative subgroup $\Theta$ of $\Gamma \backslash 0$ generated by $\bar{K},-1$ and $\delta$. For each $m>0$ we fix an equation expressing $m$ as a sum of elements from $\Theta$ as follows:

For the integer 1 we take the expression $1=1$. Having picked an expression for $m-1$, we consider the expressions for $m$ as a sum of as few terms as possible of $\Theta$, and from amongst these we pick one that has the largest number of summands in common with the expression for $m-1$. Clearly the elements of $\Theta$ occurring in these expressions are infinite in number; thus we can find an increasing sequence $\left\{m_{i}\right\}$ of positive integers and a sequence $\left\{\theta_{i}\right\}$ of elements of $\Theta$ such that $\theta_{i}$ appears in the expression for $m_{i}$ but such that $\pm \theta_{i}$ does not appear in the expression for any integer $m$ with $0<m<m_{i}$. If we subtract the equation for $m_{i}-1$ from the equation for $m_{i}$ we obtain an expression for 1 as a sum of at most $2 n_{1}$ elements of $\Theta$. In this expression we delete the summands arising from the common summands in the expressions for $m_{i}-1$ and $m_{i}$. We claim that the resulting expression for 1 has no nontrivial subsums equal to zero: if this is not the case, then the choice of expressions of minimal length for $m_{i}-1$ and $m_{i}$ implies that the subsum contains equal numbers of terms from the expressions for $m_{i}-1$ and $m_{i}$, and replacing a subsum in the expression for $m_{i}$ by a subsum from the expression for $m_{i}-1$ we can increase the number of summands that these expressions have in common. Thus we obtain infinitely many expressions for 1 as sums of at most $2 n_{1}$ elements of $\theta$, and it is clear that they are essentially distinct. However, this is a contradiction to the theorem of Evertse and Van der Poorten and Lemma 2 is proved.

5. An example. The characterization of elliptically embedded subgroups of polycyclic by finite groups given by the Theorem does not apply for soluble minimax groups. Let $p$ be an odd prime and $A$ a quasicyclic $p$-group, and let $h$ be the automorphism of $A$ which inverts every element of $A$. We define $G$ to be the split extension of $A$ by $H=\langle h\rangle$. Thus $G$ is a metabelian minimax group and $H$ is a subgroup of infinite index. Let $K$ be a subgroup of $G$. If $K \geq A$ then $G=H K$. Otherwise $K \cap A$ is finite and $K$ is finitely generated since $K /(K \cap A)$ is cyclic. Suppose that $K=\left\langle a_{1} h_{1}, \ldots, a_{r} h_{r}\right\rangle$, where $a_{i} \in A$ and $h_{i} \in H$ for each $i$. The subgroup $B=\left\langle a_{1}, \ldots, a_{r}\right\rangle$ is finite and normal in $G$, and $\langle H, K\rangle \leq H B$. It follows that $H$ has finite index in $\langle H, K\rangle$ and that $\langle H, K\rangle=(H K)^{n}$ for some $n$. Therefore $H$ is elliptically embedded in $G$. On the other hand, if $a \in A \cap N_{G}(H)$, then $[a, h] \in A \cap H=1$, so that $a$ is centralized by $h$ and $a=1$. It follows that $H=N_{G}(H)$ and that $H$ cannot be subnormal in a subgroup of finite index in $G$. 


\section{REFERENCES}

1. J. H. Evertse, On sums of $S$-units and linear recurrences, Compositio Math. 53 (1984), 225244.

2. A. H. Rhemtulla and J. S. Wilson, On elliptically embedded subgroups of soluble groups, Canad. J. Math. (to appear).

3. A. J. Van der Poorten, Additive relations in number fields, Séminaire de Théorie des Nombres de Paris (1982-1983), 259-266.

Department of Mathematics, University of Alberta, Edmonton, Alberta, CANADA

Christ's College, Cambridge, Great Britain 